\title{
Nanosponges (NSs): Using as a Nanocarrier for Anti Cancer Drug Delivery Applications
}

\author{
Ayhan BERGAL ${ }^{1 *}$, Müberra ANDAÇ² \\ 1 * Ondokuz Mayıs University, Graduate School of Natural and Applied Sciences, Department of Nanoscience and Nano- \\ technology, Phone: 05327984165, ayber2002@yaoo.com,-9104 \\ 2 Ondokuz Mayıs University, Faculty of Science and Literature, Department of Chemistry, mandac@omu.edu.tr
}

\begin{abstract}
Nanosponges (NSs) are non-toxic, porous, solid polymers, having three dimensional structures which stable at high temperature and can form inclusion complexes with many molecules due to their cavity size. NSs are prepared by reacting $\beta$-cyclodextrin $(\beta-C D)$ with cross-linkers such as pyromellitic anhydride, hexamethylene diisocyanate, carbonyl-diimidazole or diphenyl carbonate.

Many anti-cancer drugs with both hydrophilic and lipophilic properties can be loaded or integrated into NS and ultimately increase the characteristics of the drugs. The most important properties and advantages of NSs are easily synthesized and modified, provide high drug loading capacity along with controlled release, increase the solubility and bioavailability of water-soluble molecules. In addition, NSs protect the degradable actives from environmental influences, biologically safe and biodegradable compared to other nanocarriers. This review has highlighted the structure and properties of CD-NS as well as demonstrating a new generation of functionalized NS studies as an innovative drug delivery agent for pharmaceutical applications.
\end{abstract}

Keywords: Nanosponge, Cyclodextrin, Nanocarriers, Controlled Drug Delivery, Guided Drug Delivery, Cancer

\section{INTRODUCTION}

According to the data of the world health organization, cancer is defined as the second most important disease which has a fatal impact on human health of the 21st century ${ }^{1}$. Strategies developed for cancer treatment include chemotherapy, surgery, hormone therapy, radiation therapy and recently nanoparticular-based anti-cancer drugs and targeted therapy. However, traditional cancer treatment

*Corresponding Author: Ayhan Bergal, e-mail: ayber2002@yahoo.com

ORCIDS

Ayhan BERGAL: 0000-0002-5930

Müberra ANDAÇ: 0000-0001-7262-9762

(Received 27 March 2020, accepted 10 September 2020) 
methods have some disadvantages compared to innovative methods. Although it has a positive effect on the destruction of cancer cells in chemotherapy applications where cancer drugs are used, it has many negative features such as damaging normal healthy cells in the tumor environment, showing toxic properties depending on the doses used and having side effects. Surgical treatment, however, involves risks such as tumor cells invading or spreading other tissues, reoccurrence, although local cancerous tissue is removed.

In the radiation therapy that uses high energy radiation, tumor cells can be eliminated and prevent them from dividing and multiplying. During radiotherapy, the tumor area is determined and the rays are sent to the cancerous cell at a higher dose. In the treatments, a large area is used to give adequate amount of irradiation to the tumor tissue, and accordingly, healthy tissue damage and side effects can be seen much more.

Most drugs currently in use have poor biopharmaceutical properties ${ }^{2}$, low permeability, solubility and intestinal absorption, and short blood circulation times that cause various toxic side effects, therefore limiting their use in clinical applications. These adverse conditions cause rapid excretion, dose-related side effects, and deterioration in gastrointestinal fluids, in vitro instability and lack of selectivity ${ }^{3}$.In order to overcome disadvantages, drug targeting and delivery systems (DTDS) have been broadly developed, investigated and researched, especially with the aim of nano-carrier based DTDS. Treatment using nanoparticles (NPs) is known as a new type of cancer treatment method that improves the pharmacological properties of drugs and removes tumors in normal tissues with minimal effect. In this method, traditional cancer drugs are directly conjugated to NPs or integrated with a suitable ligand, and have positive results such as increased stability of the drugs, biocompatibility, and less impact on healthy cells and much less side effects in traditional methods. The accumulation of drug loaded nanostructure in the tumor cell can vary depending on the circulation time, shape, size of the structure and the targeted tumor type ${ }^{3}$.

The main function of NPs develop is the delivery and controlled release of therapeutic drugs to the targeted area. It is prominent that the nanomaterials used for this aim should be biocompatible, soluble and secure ${ }^{4-7}$. It is also important that NPs should be non-toxic, non-mutagenic, have high stability, and have the capacity to deliver therapeutic agent to specific site.

For this objectives, many nano-based drug delivery system was developed, designed and manufactured such as nanosponges, polymeric micelles, solid lipid nanoparticles, metallic nanoparticles (silver, gold), carbon nanotubes, nanogels, dendrimers, liposomes, nanocrystals, magnetic nanoparticles and micro- 
capsules. For instance, Nanosponge is a three-dimensional, solid, insoluble structure that is obtained by a variety of synthesis techniques using cyclodextrins (CD) (which may be $\alpha-C D, \beta-C D, \gamma-C D$ ) with polymeric crosslinkers. Solid lipid nanoparticles (SLNs) are structures that are used as drug delivery material that researchers work on, consisting of emulsifiers, water / solvents and lipids, and have many advantages such as low toxicity, easy production, controlled drug release, and delivering drugs of particular lipofolic nature ${ }^{8}$.

Dendrimers are polymers containing a large number of branched monomers consisting of a central core. These structures, which have many functional groups thanks to the branched monomers composed of dendrons, are used as drug delivery agents due to their compatibility and flexible structures. Denimers synthesized from many polymers such as polyamidoamine PAMAM), polypropyleneimine (PPI) have nanoparticle drug delivery potential due to their biological compatibility. In addition, these structures are used for photothermal therapy gene therapy applications ${ }^{9,10}$.

The liposomes, consisting of small spherical vesicles, are two-layer structures made of phospholipids containing a hydrophobic hydrocarbon chain and a hydrophilic head, and are used as drug delivery agents. Due to their high compatibility, liposomes, which can be formed in nanometer or micrometer size due to their single or multi-layer structure, can carry structures such as medicine, protein, antigen, DNA and can be used in other therapies as well as cancer therapy ${ }^{11}$.

However, due to the disadvantages of NPs, it is difficult to develop an optimal drug delivery system that achieves the desired result, some nanomaterials are difficult to apply as a drug delivery agents due to their degradation or toxicity effects in the body parts, and this requires sufficient time and research. For instance, the disadvantage of solid lipid nanoparticles is that SLNp have a crystalline structure that causes drug release due to unexpected polymeric degradation ${ }^{8}$. Dendrimers, on the other hand, may show toxic properties due to the presence of peripheral amine carboxylate, phosphonate, sulfonate groups, as well as the fact that they can show non-specific drug delivery and sudden and uncontrolled drug release due to its open network ${ }^{12}$.

Liposomes also have disadvantages such as low content of drug loading, high cost, poor stability and undesired hydrophobic drug release.

Due to their characteristic structure and properties, NPs can be passively, actively or physically delivered to the target site, e.g. tumor cell ${ }^{13-16}$. Targeting the NPs to the tumor region depends on the structural feature of the tumor tissue, that is, the leaky vascular feature and the weak excretory system, which is called 
enhanced permeability and retention (EPR) effect. The nanostructure caused by the EPR effect accumulates in the tumor region and this is defined as passive targeting. The NPs whose surface is modified with ligands such as peptides or antibodies binds by recognizing reproducers on the cell surface and internalized into the cell through endocytosis, leaving the loaded drugs. This event is defined as active targeting. On the other hand, accumulation of nanostructure can be performed in tumor tissues through external effects such as ultrasound, magnetic field, heat, light waves (UV, infrared) ${ }^{17-18}$.

Again according to the designed NPs structure, internal stimulatory mechanisms (pH-guided ${ }^{19}$, enzymes ${ }^{20}$, temperature ${ }^{21}$ ) or as external stimuli (electric field ${ }^{22}$, the magnetic field ${ }^{23}$, Light ${ }^{24}$, voltage excitations ${ }^{25}$ ultrasound ${ }^{26}$, and other chemicals ${ }^{27-29}$ ) may release the drug in the cell.

In researches on some nanoparticles used for drug delivery strategies, the solubility and effectiveness of drugs have been increased and it has been proven that these structures can be used as effective delivery agents. For instance, dendrimers can be used as delivery agents for non-steroidal anti-inflammatory lyophilic drugs. For example, in a study, the drug containing the amino group was encapsulated into PPI or PAMAM type dendrimers and its solubility was increased ${ }^{12,30}$.

In a study with solid lipid nanoparticles, tamoxifen loaded SLNp was prepared and its effectiveness on MCF7 cancer cells was investigated. In in vitro cytotoxide research, they emphasized that the efficiency of tamoxifen loaded SLNp increased when compared to free tamoxifen ${ }^{31,32}$.

\section{METHODOLOGY}

\section{Cyclodextrin Based Nanosponges (CD-NSs)}

Nanosponges are effective nano-carriers that researchers focus on as a drug delivery agent, which has recently attracted the attention of many researchers due to their characteristic structures. NSs have been developed to eliminate the disadvantages of the traditional medicines described in detail above and increase the effectiveness of drugs. Briefly, Nanosponges are obtained by using cyclodextrins (CD) ( $\alpha-C D, \beta-C D, \gamma-C D)$ with polymeric crosslinkers (such as diphenyl carbonate). Since the main component is cyclodextrins, it is useful to mention the definition and structure of CDs.

\section{Chemical Structure and Properties of CDs}

Cyclodextrin (CD) is defined as a water-soluble, biocompatible cyclic oligisaride, which is formed by the enzymatic degradation of starch, elementary polysaccharides in nature, by the Bacillus amylobacter ${ }^{33}$ bacterium, and con- 
tain a hydrophilic outer surface and a lipophilic cavity that is formed by the $\alpha-1,4$ glycosidic binding of D-glycosupranoses (Figure 1.). $\beta-C D, \gamma-C D, \alpha-C D$ are named depending on the number of glucosupranose units in their structure. For instance, the structure formed by connecting 7 glucosupranose units with glycosidic bond is called $\beta$-CD (Figure 1.).

Despite the fact that CDs in higher structures are found in nature, the difficulty of obtaining and being able to form an inclusion complex with very few substances were not preferred in terms of research. Among them, $\beta-\mathrm{CD}$ is the most preferred and profound studied structure because its convenient cavity, which can interact with many inorganic and organic substances, in addition, ensures high drug loading capacity, easy availability and affordability.

Since 1970, CDs have been used in medicine, food, cosmetics, textiles, catalysts, biotechnology, and more recently as a drug targeting and delivery system in pharmacology, nanotechnology and the pharmaceutical industry.

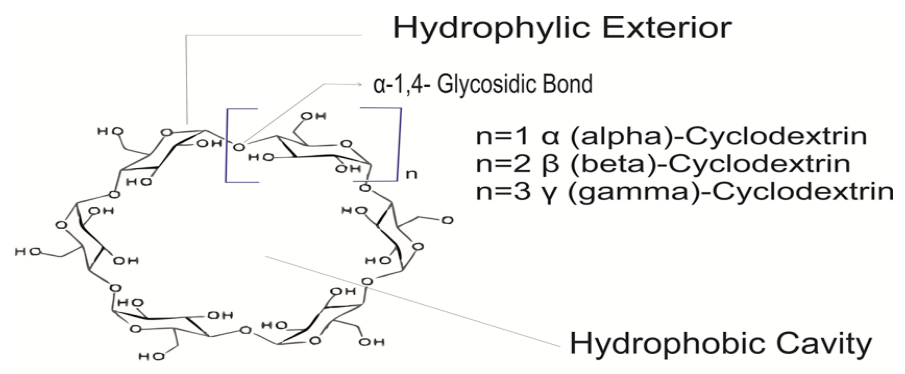

Figure 1. Dimensions and chemical structure for $\alpha-, \beta$ - and $\gamma-C D(n=6,7$ and 8 , respectively).

\section{Cyclodextrin-Based Nanosponge (CD-NS) Concept}

Natural CDs do not form inclusion complexes with particular drugs which has high molecular weight or hydrophilic property, in addition some CDs limit their use since they show toxic properties when injected intravenously ${ }^{34-36}$. Therefore, many chemical modification studies have been carried out in order to use them as a nano drug carrier system, eliminate the disadvantages of CDs, and improve their physical, chemical properties. One of these alterations is the formation of NSs by reacting natural CDs with the crosslinkers described below. In 1998, DeQuan Li and Min Ma were the first researchers to use the term cyclodextrin-based Nanosponges ${ }^{37}$ and develop the NS obtained by bonding $\beta$-CD with a cross-linker (such as diisocyanate) for the treatment and purify of wastewater ${ }^{38}$. In their research, they showed that CD- NSs completely removed wastes such as p-chlorophenol from wastewater at even a billionth level. 
However, the development of CD-based NSs by reacting of natural CDs with crosslinkers and the application of NSs as a nano drug delivery system was first demonstrated by Trotta and his team ${ }^{39-41}$.

Nanosponge's are defined as nanoscale hyper-crosslinked polymers which is non-toxic, stable at high temperature, spongy pores and a three-dimensional network structure. It is synthesized by using main component of $\beta, \alpha$, or $\gamma$-cyclodextrins and crosslinked by proper amount of crosslinkers (such as pyromellitic anhydride, hexamethylene diisocyanate, carbonyl-diimidazole, diphenyl carbonate, (Figure 2,3.) NSs can preserve the hydrophilic and hydrophobic drugs in their three dimensional cavity. It is usually prepared from $\beta$-cyclodextrins compared with other native $\operatorname{CDs}(\alpha$, and $\gamma$ ) because $\beta$-CD has the highest drug encapsulation sites, higher complexes and stability due to the appropriate cavity size ${ }^{39,40}$.

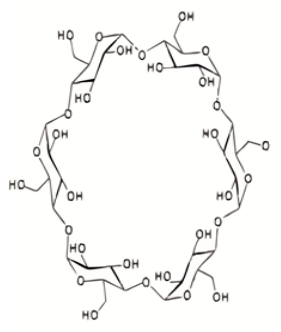

Cyclodextrin

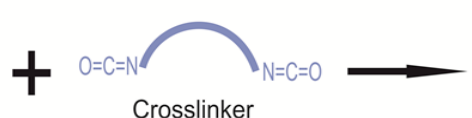

Crosslinker

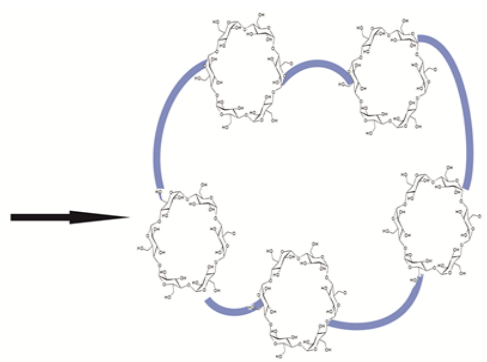

Cyclodextrin Nanosponge

Figure 2. Cyclodextrins bind to the crosslinking polymer to form NanospongeThe four most basic cross-linking polymers are used in the synthesis of NSs with appropriate synthesis methods, such as anhydride crosslinkers, carbonyl crosslinkers, diisocyanate linkers, and epichlorohydrin (Figure 3.). 


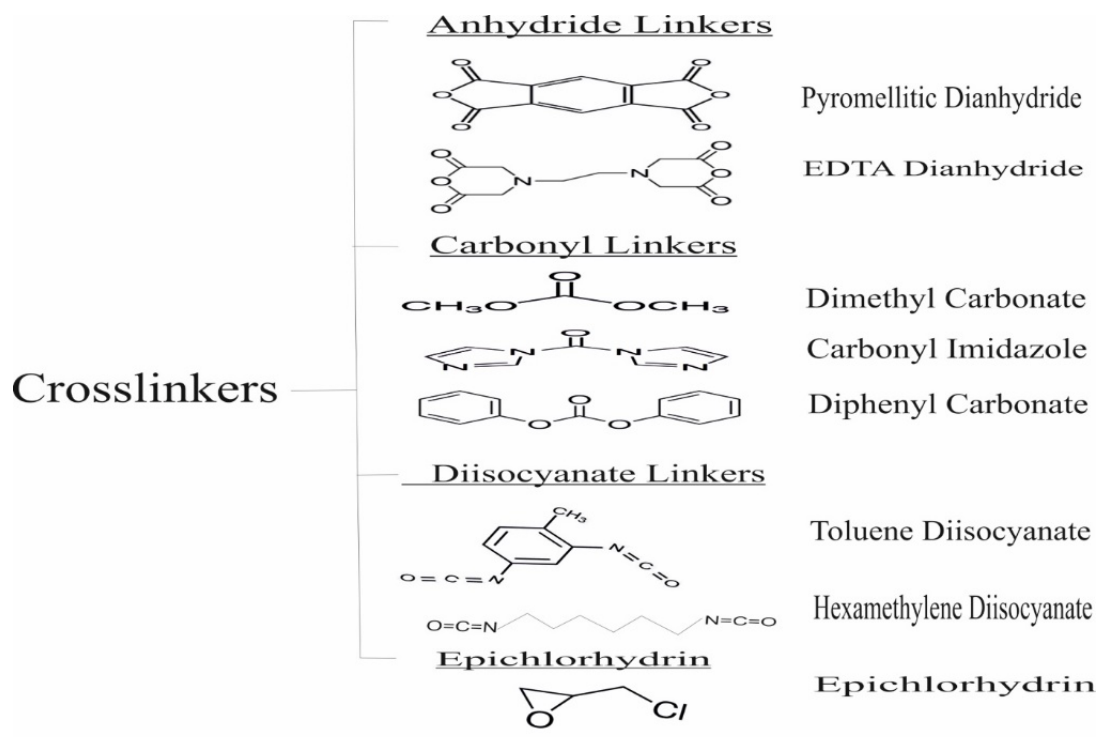

Figure 3. Common crosslinkers used in Nanosponge synthesis.

\section{RESULTS and DISCUSSION}

\section{Methods Used in Nanosponge Synthesis}

Melting method ${ }^{42}$, solvent method ${ }^{43}$, ultrasound assisted synthesis ${ }^{42}$ microwave-assisted synthesis 44,45 methods are used to obtain desired characteristics and different sizes of NS. In the melting method, briefly, the crosslinker such as diphenyl carbonate and beta cyclodextrin in certain molar ratios are melted and gradually heated to a temperature of $90-100{ }^{\circ} \mathrm{C}$ under magnetic stirring, and the by-product formed is removed and cooled at room temperature. The solid product (Figure 4.) formed is washed with distilled water and acetone, and the unreacted product is removed ${ }^{42}$.

In solvent method, cyclodextrin is dissolved in a polar solvent such as dimethylsulfoxide and reacted with a crosslinker such as carbonyldiimidazole at a certain molar ratio for about 4-5 hours at $90{ }^{\circ} \mathrm{C}$. When the reaction is over, an excess of water is added to remove the unreacted crosslinker and the solid is obtained by filtration. Then Sohxlet- extracted with acetone to remove from by-products (Figure 4.) $43,44,46$.

In ultrasound assistand synthesis, cyclodextrin and cross-linker are put in a bottle in a solvent-free environment and subjected to sonication in the ultrasound bath for approximately 5 hours at $90^{\circ} \mathrm{C}$. After cooling at room temperature, processes are performed as specified in the solvent method ${ }^{39,42}$. 
The product obtained by the solvent method has a spherical morphology and a high dissolution power for water-soluble molecules. In addition, submicron spherical nanosponges can be obtained by ultrasound assisted synthesis using a suitable crosslinker.

Microwave assisted synthesis is known as the easiest method in obtaining cyclodextrin based nanosponge compared to other methods. The method is used to obtain homogeneous crystallinity of NSs as well as a synthesis method which reduces reaction time four times compared to melting method.

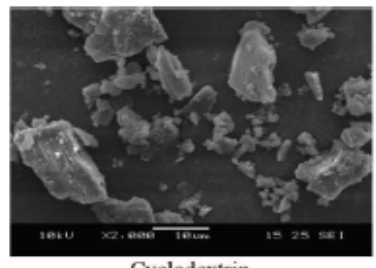

Cyclodextrin

a

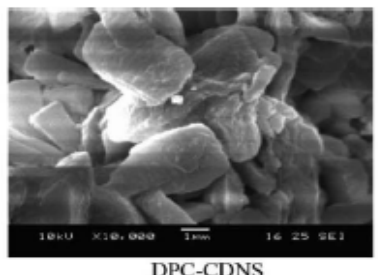

$\mathrm{b}$

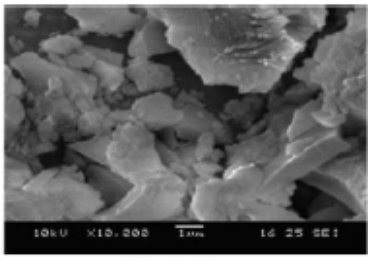

PMDA-CDNS

$\mathrm{C}$

Figure 4. SEM image of a) Cyclodextrin b) Diphenyl Carbonate crosslinked NS c) Pyromellitic Anhydride crosslinked NS ${ }^{46}$

\section{Factors Affecting Formation of NSs}

There are also important factors affecting the formation of NS. These are:

Drug Type: Besides the fact that the drugs used are hydrophilic or hydrophobic, the properties of the drugs known as 'Five rules of Lipinski' affect the formation of NSs.

Polymer Type and Crosslinkers: The type of CD (being $\alpha-, \beta$ - and $\gamma$-CD) and the crosslinkers used play an important role in NSs formation. Specifically, the determination of $\mathrm{CD}$ and cross-linker molar ratios causes the particle size of NS to form in the desired size; however, in the cross-linker types play an important role in transforming NSs into a three-dimensional structure suitable for hydrophilic or hydrophilic drugs.

Temperature: Increasing the temperature, for example, can especially lead to a decrease or weakening of intermolecular hydrophobic forces (such as Vander waals force) between NSs and the drug ${ }^{47}$.

Nanosponge Preparation Method: The effectiveness of the method used is important in terms of both the formation of NS and drug coplex and integration of the drug into NS, depending on the nature of the drug and polymer used in the formulation. One of the most useful techniques for the drug / nanosponge complex is the freeze-drying technique ${ }^{48}$. 
Degree of Substitution: The ability to form nanosponge complexes may vary depending on the number, position and structure of the substituent on the parent polymer. For example, the higher the degree of substitution on the parent polymer, the more cross-linking can take place between CD and crosslinker ${ }^{49}$.

\section{Nanosponge Toxicity}

Toxicity studies are necessary studies that are extremely important in terms of whether or not the dose of the drug used and the nanocarrier designed for drug delivery is toxic to humans and animals, hence to evaluate the usability of the structure.

Toxicity studies with NS have shown no toxic effects or adverse effects. For example, by injecting NS onto Swiss albino mice, the acute systemic toxicity of NS has been shown to be safe from 500 to $5000 \mathrm{mg} / \mathrm{kg}$ and does not show any signs of toxicity or adverse reactions ${ }^{50}$. In addition, the study of oral administration of nanosponge was tested in mice ${ }^{51}$ without significant side effects. In vitro toxicity studies using cell cultures such as MCF7, COS, HELA using the MTT test also showed that NSs had no cytotoxic effect ${ }^{39}$.

\section{The Most Common General Applications of Nanosponge}

Due to their sophisticated structure and biocompatibility, they can deliver hydrophobic and a hydrophilic drug, provide controlled release, enhance the satiability of drugs as well as increases the dissolution rate of drugs in aqueous environments. NSs can be prepared and used in the pharmaceutical field as capsules or tablets, parenterally, topically in hydrogel forms and as adjuvants as well as in applications such as delivery and targeting of drugs ${ }^{41,52-54}$.

To summarize a few of the general drug delivery application areas of NSs,

Increasing solubility of drugs: Shende et al. ${ }^{54}$ researched the effect of the low-soluble meloxicam drug on the stability, solubility, slow and sustained release of the inclusion complex formed with $\beta-\mathrm{CD}$ alone and NS.

For this goal, 1: 1 molar ratio of $\beta$-CD with meloxicam drug and 1: 8 molar ratio of NS obtained by using $\beta$-CD and pyromellitic dianhydride cross-linker (PMDA). Meloxicam, which has a solubility of $9.45 / \mathrm{g} / \mathrm{ml}$ in aqueous medium alone has increased to $19.07 \mathrm{~g} / \mathrm{ml}$ after combining with $\beta$-CD, and this value has increased to $36.61 \mathrm{~g} / \mathrm{ml}$ in integration with $\beta$-CD based NS. Therefore, they have successfully proved that NS increases the solubility of the drug.

Controlled and sustained drug release: Cavalli et al..$^{55}$ has obtained NS by using beta cyclodextrin and DFC crosslinker and integrates 3 different type of drugs with both hydrophilic (eg Doxorubicin) and lipophilic (eg flurbiprofen or dexamethasone) property to NS, and conducted a research to examine drug 
solubility and its effect on drug release profile.

Effect of NS study has indicated that the solubility increases about $4 \%$ by weight for doxorubicin and $15 \%$ by weight for lipophilic drugs. Compare to release profile, doxorubicin is released very slowly in the physiological medium at pH 1.1, but it is faster when the $\mathrm{pH}$ is increased to 7.4. In addition, they demonstrated the interaction between the drug and nanosponge and sustaine drug release over time, emphasizing that drug release from NSs was less than $20 \%$ for dexamethasone and for flurbiprofen less than 10\% after 2 hours.

Protecting drugs from light or distortion: S. Anandam et al..$^{56}$ conducted discrete irradiation tests for complex quercetin and pure quercetin at the equal concentration so the protective effect of NS on photodegradation of quercetin investigated. As a result, they have proved that pure quercetin disorder is much faster than NS encapsulated quercetin. In addition to this study, they proved that concentration of pure quercetin decreased by more than $50 \%$ in simulated intestinal fluid (SIF) within 6 hours, but NS encapsulated formulation did not show significant reduction, therefore NS proved that it effectively protects drugs from photodegradation. In addition, they emphasized that the antioxidant activity of quercetin and the radical scavenging activity of DPPH increased 569 times with the NS complex. ${ }^{56}$.

Protein and enzyme transport: Bovine has been used as a model protein and has been shown to increase protein stability by incorporating serum albumin into poly amidoamine cross-linked NS ${ }^{57}$.

As oral and topical drug delivery systems: Gangadharappa, H. V. et al..$^{8}$ conducted a research on pharmacokinetics and skin irritation on mice for the topical application of Nanasponges by integrating the water-soluble lipophilic celecoxib drug with NS formed in hydrogel. They emphasized that the optimized formulation NS-4 does not irritate the skin on mice, nanosponge hydrogels obtained can be used as topical drug release for celecoxib, as well as meeting the requirements in human use in this dosage form ${ }^{58}$.

As a gas delivery agent: Nanosponge formulations have demonstrated the ability to function and store as a reservoir for large quantities of various gas types such as carbon dioxide and oxygen ${ }^{59}$. In addition, NSs are used as cosmetics ${ }^{60}$, agricultural ${ }^{61}$ and water treatment ${ }^{62}$.

Recently, researchers have been focusing on designing functionalized NS by surface modification of NSs and enhancing intracellular cell interaction by using ligands. In a study conducted for this purpose, NS was synthesized with $\beta$-CD and diphenyl carbonate (DPC) crosslinker and functionalized the surface of NS with hydrogen succinate (CHS) to investigate cellular interaction and 
protein binding by adding cholesterol ${ }^{63}$. In the study used doxorubicin DOX as a model drug, the cellular binding activity revealed an increase in cellular uptake and Dox adsorption of Dox-loaded $\beta$-CD-NS using the surface functionalization approach. They emphasized that they show preferable internalization in cells due to the interaction among cell membrane and CHS. In the same study, in vitro cytotoxicity of surface-modified $\beta$-CD-NS was investigated in the HeLa cell line and the data obtained showed that surface-modified $\beta$-CD-NS samples were biologically friendly and did not show significant toxicity in HeLa cells ${ }^{63}$.

Drug targeting provides significant advantages in terms of drug delivery and drug release compared to traditional treatment methods, as well as significant potential advantages such as reducing the side effects of drugs, increasing their bioavailability and biocompatibility (thus encapsulating lipophilic and / or hydrophilic materials and keeping them in the blood for a long time and in a controlled manner). In addition, it is a promising nanocarrier that can increase the bioavailability and aqueous solubility by protecting the drugs from degradation in the physiological environment, which may result in increased specificity with yield when used with ligands for targeting.

There are many more things to discover, research and develop for NS. For example, obtaining functionalized NS, integrating ligands into NS, delivering the nanostructure to the targeted region through active or passive mechanisms and binding to the cell receptors. In addition, many studies are needed, such as intracellular uptake and drug release using internal and external stimulants, to integrate NS with metallic or inorganic nanostructures to increase drug loading capacity. However, functionalized NS surface engineering studies are limited to several publications.

In addition to increasing the efficiency and specificity of the targeted regions and reducing the toxicity of the drug, many researches are needed to increase the effect level of the drug by taking the drug release slowly and gradually.

However, mathematical modeling of NS integration into the cell and conducting positively charged NS studies to overcome the problems encountered for gene or nucleic acid delivery may be considered as future approaches.

\section{CONFLICT OF INTEREST}

The authors have not declared any personal or financial conflicts of interest with any other parties or organizations.

\section{ACKNOWLEDGMENT}

The author thanks to Prof. Müberra ADAÇ for its technical support and supervision. 


\section{REFERENCES}

1. Health topics https://www.who.int/health-topics/ (accessed Mar 20, 2020).

2. Siegel, R.; Ma, J.; Zou, Z.; Jemal, A. Cancer statistics. CA: A Can. J. For Clinic. 2014, 64, 9-29.

3. Valente, F.; Astolfi, L.; Simoni, E.; Danti, S.; Franceschini, V.; Chicca, M.; Martini, A. J. Drug Del. Sci. and Tech. 2017, 39, 28.

4. Torchilin, V. P. Drug targeting. Eu. J. Pharmaceut. Sci. 200o, 11.

5. Jemal, A.; Bray, F.; Center, M. M.; Ferlay, J.; Ward, E.; Forman, D. Global cancer statistics. CA: A Can. J. For Clinic. 2011, 61, 69-90.

6. Chen, Z. (G. Small-molecule delivery by nanoparticles for anticancer therapy. Trends in Mole. Med. 2010, 16, 594-602.

7. Jin, S.-E.; Jin, H.-E.; Hong, S.-S. Targeted Delivery System of Nanobiomaterials in Anticancer Therapy: From Cells to Clinics. BioMed Res. Int. 2014, 2014, 1-23.

8. Amoabediny, G.; Haghiralsadat, F.; Naderinezhad, S.; Helder, M. N.; Kharanaghi, E. A.; Arough, J. M.; Zandieh-Doulabi, B. Int. J. Poly. Mater. and Poly. Biomater. 2017, 67 (6), 383.

9. Wang, H.; Huang, Q.; Chang, H.; Xiao, J.; Cheng, Y. Biomater. Sci. 2016, 4 (3), 375.

10. Cai, X.; Zhu, H.; Zhang, Y.; Gu, Z. ACS App. Mater. \& Interfaces 2017, 9 (11), 9402.

11. Yingchoncharoen, P.; Kalinowski, D. S.; Richardson, D. R. Pharmaco. Rev. 2016, 68 (3), 701.

12. Patel, V.; Rajani, C.; Paul, D.; Borisa, P.; Rajpoot, K.; Youngren-Ortiz, S. R.; Tekade, R. K. Drug Del. Sys.2020, 333.

13. Colombo, P. Opinion Paper: Modular Drug Delivery Systems for Personalized Oral Dosage Forms. R. Pat. on Drug Del. \& Form. 2016, 10, 3-3.

14. Maeda, H.; Nakamura, H.; Fang, J. The EPR effect for macromolecular drug delivery to solid tumors: Improvement of tumor uptake, lowering of systemic toxicity, and distinct tumor imaging in vivo. Ad. Drug Del. Rev. 2013, 65, 71-79.

15. Decuzzi, P.; Godin, B.; Tanaka, T.; Lee, S.-Y.; Chiappini, C.; Liu, X.; Ferrari, M. Size and shape effects in the biodistribution of intravascularly injected particles. J. Cont. Re. 2010, 141, $320-327$.

16. Jeong, S.-Y.; Woo; Chung; Jung; Ju; Kang; Lee; Seo; Lee; Lee; et al. Preclinical evaluation of injectable sirolimus formulated with polymeric nanoparticle for cancer therapy. Int. J. Nanomed. 2012, 2197.

17. Bidram, E.; Esmaeili, Y.; Ranji-Burachaloo, H.; Al-Zaubai, N.; Zarrabi, A.; Stewart, A.; Dunstan, D. E. J. Drug Del. Sci. and Tech. 2019, 54, 101350.

18. Chertok, B.; Langer, R. Theranostics 2018, 8 (2), 341.

19. Chen, Y.-C.; Liao, L.-C.; Lu, P.-L.; Lo, C.-L.; Tsai, H.-C.; Huang, C.-Y.; Wei, K.-C.; Yen, T.-C.; Hsiue, G.-H. The accumulation of dual $\mathrm{pH}$ and temperature responsive micelles in tumors. Biomaterials 2012, 33, 4576-4588.

20. Lee,Y.;Miyata, K.; Oba, M.; Ishii, T.; Fukushima, S.; Han, M.; Koyama, H.; Nishiyama,N.; Kataoka,K. Charge-Conversion Ternary Polyplex with EndosomeDisruption Moiety:ATechnique for Efficient and Safe Gene Delivery. Angewandte Chemie Int. Ed. 2oo8, $47,5163-5166$. 
21. Zhang, J.; Feng, K.; Cuddihy, M.; Kotov, N. A.; Ma, P. X. Spontaneous formation of temperature-responsive assemblies by molecular recognition of a $\beta$-cyclodextrin-containing block copolymer and poly(N-isopropylacrylamide). Soft Matter 2010, 6, 610-617.

22. Ge, J.; Neofytou, E.; Cahill, T. J.; Beygui, R. E.; Zare, R. N. Drug Release from ElectricField-Responsive Nanoparticles. ACS Nano 2011, 6, 227-233.

23. Namiki, Y.; Namiki, T.; Yoshida, H.; Ishii, Y.; Tsubota, A.; Koido, S.; Nariai, K.; Mitsunaga, M.; Yanagisawa, S.; Kashiwagi, H.; et al. A novel magnetic crystal-lipid nanostructure for magnetically guided in vivo gene delivery. Nature Nanotech. 2009, 4, 598-606.

24. Chen, J.; Guo, Z.; Wang, H.-B.; Gong, M.; Kong, X.-K.; Xia, P.; Chen, Q.-W. Multifunctional Fe3O4@C@Ag hybrid nanoparticles as dual modal imaging probes and near-infrared light-responsive drug delivery platform. Biomaterials 2013, 34, 571-581.

25. Chakravarty, P.; Qian, W.; El-Sayed, M. A.; Prausnitz, M. R. Delivery of molecules into cells using carbon nanoparticles activated by femtosecond laser pulses. Nature Nanotech. 2010, 5 , 607-611.

26. Stanley, S. A.; Gagner, J. E.; Damanpour, S.; Yoshida, M.; Dordick, J. S.; Friedman, J. M. Radio-Wave Heating of Iron Oxide Nanoparticles Can Regulate Plasma Glucose in Mice. Science 2012, 336, 604-608.

27. Korin, N.; Kanapathipillai, M.; Matthews, B. D.; Crescente, M.; Brill, A.; Mammoto, T.; Ghosh, K.; Jurek, S.; Bencherif, S. A.; Bhatta, D.; et al. Shear-Activated Nanotherapeutics for Drug Targeting to Obstructed Blood Vessels. Science 2012, 337, 738-742.

28. Takae, S.; Miyata, K.; Oba, M.; Ishii, T.; Nishiyama, N.; Itaka, K.; Yamasaki, Y.; Koyama, H.; Kataoka, K. PEG-Detachable Polyplex Micelles Based on Disulfide-Linked Block Catiomers as Bioresponsive Nonviral Gene Vectors. J. Am. Chem.Soc. 2008, 130, 6001-6009.

29. Broaders, K. E.; Grandhe, S.; Fréchet, J. M. J. A Biocompatible Oxidation-Triggered Carrier Polymer with Potential in Therapeutics. J. Am. Chem.Soc. 2011, 133, 756-758.

30. Markowicz-Piasecka, M.; Mikiciuk-Olasik, E. Nanobiomater. in Drug Del. 2016, 39.

31. Eskiler, G. G.; Cecener, G.; Dikmen, G.; Egeli, U.; Tunca, B. Eu. J. Pharmaceut. Sci. 2o18, 120,73 .

32. Daima, H. K.; PN, N.; Ranjan, S.; Dasgupta, N.; Lichtfouse, E. Nanoscience in medicine; Springer: Cham, Switzerland, 2020.

33. Jansook, P.; Ogawa, N.; Loftsson, T. Cyclodextrins: structure, physicochemical properties and pharmaceutical applications. Int. J. Pharmaceut. 2018, 535, 272-284.

34. Davis, M. E.; Brewster, M. E. Cyclodextrin-based pharmaceutics: past, present and future. Nature Rev. Drug Del. 2004, 3, 1023-1035.

35. Loftsson, T.; Jarho, P.; Másson, M.; Järvinen, T. Cyclodextrins in drug delivery. Ex. Op.on Drug Del. 2005, 2, 335-351.

36. Gray, J. E.; Weaver, R. N.; Purmalis, A. Ultrastructural Observations of Chronic Progressive Nephrosis in the Sprague-Dawley Rat. Vet. Pathology 1974, 11, 153-164.

37. Li, D.; Ma, M. Cyclodextrin polymer separation materials, 1998, WO 9822197.

38. Li, D.; Ma, M. Nanosponges For Water Purification. Clean Prod. and Process. 2ooo, 2, 0112-0116.

39. Trotta, F.; Zanetti, M.; Cavalli, R. Cyclodextrin-based nanosponges as drug carriers. Beilstein J. Org. Chem. 2012, 8, 2091-2099. 
40. Trotta, F. Cyclodextrin Nanosponges and their Applications. Cyclodextrins in Pharma., Cos., and Biomed. 2011, 323-342.

41. Swaminathan, S.; Cavalli, R.; Trotta, F. Cyclodextrin-based nanosponges: a versatile platform for cancer nanotherapeutics development. Wiley Interdisciplinary Reviews: Nano. and Nanobiotech. 2016, 8, 579-601.

42. Inamuddin; Asiri, A. M.; Mohammad, A. Applications of nanocomposite materials in drug delivery; Woodhead Pub., an imprint of Elsevier: Oxford, 2018.

43. Osmani, R. A. M.; Bhosale, R. R.; Hani, U.; Vaghela, R.; Kulkarni, P. K. Cyclodextrin Based Nanosponges: Impending Carters in Drug Delivery and Nanotherapeutics. C. Drug Therap. 2015, 10, 3-19.

44. Singireddy, A.; Pedireddi, S. R.; Nimmagadda, S.; Subramanian, S. Beneficial effects of microwave assisted heating versus conventional heating in synthesis of cyclodextrin based nanosponges. Mater. Today: Proceedings 2016, 3, 3951-3959.

45. Anandam, S.; Selvamuthukumar, S. Optimization of microwave-assisted synthesis of cyclodextrin nanosponges using response surface methodology. J. Porous Mater. 2014, 21, 1015-1023.

46. Pushpalatha, R.; Selvamuthukumar, S.; Kilimozhi, D. Cross-Linked, Cyclodextrin-Based Nanosponges For Curcumin Delivery - Physicochemical Characterization, Drug Release, Stability And Cytotoxicity. J. Drug Del. Sci. and Tech. 2018, 45, 45-53.

47. Sehgal, N.; N, V. G.; Kanna, S. A Review On Nanosponges A Review On Nanosponges: A Boon To Targeted Drug Delivery For Anticancer Drug. Asian J. Pharmaceut. and Clinic. Re. 2019, 1-7.

48. Bhowmik, H.; Venkatesh, D. N.; Kuila, A.; Kumar, K. H. Nanosponges: A Review. Int. J. Appl. Pharmaceut. 2018, 10, 1.

49. Pandey, P.; Purohit, D.; Dureja, H. Nanosponges -A Promising Novel Drug Delivery System. Rec. Pat. on Nanotech. 2018, 12, 180-191.

50. Manakker, F. V. D.; Vermonden, T.; Nostrum, C. F. V.; Hennink, W. E. Cyclodextrin-Based Polymeric Materials: Synthesis, Properties, and Pharmaceutical/Biomedical Applications. Biomacromole. 2009, 10, 3157-3175.

51. Shende, P.; Kulkarni, Y. A.; Gaud, R.; Deshmukh, K.; Cavalli, R.; Trotta, F.; Caldera, F. Acute and Repeated Dose Toxicity Studies of Different $\beta$-Cyclodextrin-Based Nanosponge Formulations. J. Pharmaceut. Sci. 2015, 104, 1856-1863.

52. Minelli, R.; Cavalli, R.; Ellis, L.; Pettazzoni, P.; Trotta, F.; Ciamporcero, E.; Barrera, G.; Fantozzi, R.; Dianzani, C.; Pili, R. Nanosponge-encapsulated camptothecin exerts anti-tumor activity in human prostate cancer cells. Eu. J. Pharmaceut. Sci. 2012, 47, 686-694.

53. Daga, M.; Ullio, C.; Argenziano, M.; Dianzani, C.; Cavalli, R.; Trotta, F.; Ferretti, C.; Zara, G. P.; Gigliotti, C. L.; Ciamporcero, E. S.; et al. GSH-targeted nanosponges increase doxorubicininduced toxicity "in vitro" and "in vivo" in cancer cells with high antioxidant defenses. Free. Rad. Bio. and Med. 2016, 97, 24-37.

54. Shende, P. K.; Gaud, R.; Bakal, R.; Patil, D. Effect of inclusion complexation of meloxicam with $\beta$-cyclodextrin- and $\beta$-cyclodextrin-based nanosponges on solubility, in vitro release and stability studies. Colloids and Surfaces B: Biointerfaces 2015, 136, 105-110.

55. Cavalli, R.; Trotta, F.; Tumiatti, W. Cyclodextrin-based Nanosponges for Drug Delivery. J. Inclu. Pheno. and Macrocyc. Chem. 2006, 56, 209-213. 
56. Anandam, S.; Selvamuthukumar, S. Fabrication of cyclodextrin nanosponges for quercetin delivery: physicochemical characterization, photostability, and antioxidant effects. J. Mater. Sci. 2014, 49, 8140-8153.

57. Swaminathan, S.; Cavalli, R.; Trotta, F.; Ferruti, P.; Ranucci, E.; Gerges, I.; Manfredi, A.; Marinotto, D.; Vavia, P. R. In vitro release modulation and conformational stabilization of a model protein using swellable polyamidoamine nanosponges of $\beta$-cyclodextrin. $J$. Inc. Phenom. and Macrocyc. Chem. 2010, 68, 183-191.

58. Gangadharappa, H.; Prasad, S. M. C.; Singh, R. P. Formulation, in vitro and in vivo evaluation of celecoxib nanosponge hydrogels for topical application. J. Drug Del. Sci. and Tech.2017, 41, 488-501.

59. Cavalli, R.; Akhter, A. K.; Bisazza, A.; Giustetto, P.; Trotta, F.; Vavia, P. Nanosponge formulations as oxygen delivery systems. Int. J. Pharmaceut. 2010, 4O2, 254-257.

6o. Sapino, S.; Carlotti, M. E.; Cavalli, R.; Ugazio, E.; Berlier, G.; Gastaldi, L.; Morel, S. Photochemical and antioxidant properties of gamma-oryzanol in beta-cyclodextrin-based nanosponges. J. Inc. Pheno. and Macrocyc. Chem. 2012, 75, 69-76.

61. Lien, N.; Telford, J. An investigation of the inclusion complex of cyclomaltoheptaose ( $\beta$-cyclodextrin) with N-methylanthranilic acid in the solid state. Carbohyd. Re. 2009, 344, 2606-2608.

62. Euvrard, É.; Morin-Crini, N.; Druart, C.; Bugnet, J.; Martel, B.; Cosentino, C.; Moutarlier, V.; Crini, G. Cross-linked cyclodextrin-based material for treatment of metals and organic substances present in industrial discharge waters. Beilstein J. Org. Chem. 2016, 12, 1826-1838.

63. Singh, P.; Ren, X.; Guo, T.; Wu, L.; Shakya, S.; He, Y.; Wang, C.; Maharjan, A.; Singh, V.; Zhang, J. Biofunctionalization of $\beta$-cyclodextrin nanosponges using cholesterol. Carbohydr. Polym. 2018, 19o, 23-30. 